\title{
One size does not fit all for Canadian trauma systems
}

\author{
Robert S. Green, MD, BSc, DABEM ${ }^{* \neq \ddagger}$; Natalie Yanchar, MD, $\mathrm{MSc}^{\S}$
}

For over 3 decades, Canadian physicians, surgeons, paramedics, and nurses have worked with the government at the local, provincial, and federal levels to improve the outcomes of injured patients. Our trauma systems have been informed by systems in the United States and other countries, despite differences in healthcare structures. For the most part, early influencers of trauma care in Canada were predominantly surgeons with system and institutional standards and guidelines developed by organizations such as the American College of Surgeons Committee on Trauma (ACS-COT).

Recognizing the multidisciplinary nature of caring for the injured, groups such as the Trauma Association of Canada have adopted principles that reflect the value of diversity in knowledge and expertise, with the overall goal of improving patient outcomes. As such, the provision of excellence in care during trauma resuscitation in the emergency department is reliant not on role designation, but on comfort and skill in rapid decision-making and intervention. In Canada, trauma care is not driven by turf wars or patient ownership, but by what is best for the patient.

For optimal trauma system development, unique elements must be considered. These include population, geography, resources, sustainability, and the availability of specialized skill sets. Importantly, Canadian healthcare has distinct challenges that vary from coast to coast. Today, more than ever, creative solutions are required to provide care for trauma patients. When it comes to trauma systems, one size does not fit all.

In this issue of CFEM, two studies have provided valuable insights into trauma resuscitation and patient care following hospital admission via different models of care. Hayre et al. have challenged the belief that the resuscitation of major trauma patients by a "formal" trauma team leads to better outcomes when compared to care led by emergency physicians with consultations to other specialities resources as needed. ${ }^{1}$ When stratified by injury severity, the authors found little difference between the two models for most of the outcomes evaluated, with the exception of a reduced time to computed tomography (CT) and to the operating room (if needed) in the trauma team model. Despite being limited to population-level data (as opposed to patient-level data), this study should be viewed as an important initial investigation that demonstrates the value of flexibility in trauma care. The overall $88 \%$ to $89 \%$ survival rate of the two patient cohorts matches favourably to that of the National Trauma Registry (88\%), ${ }^{2}$ suggesting that models of care based on local drivers have value. Interestingly, emergency physicians performed the role of trauma team leader in the majority of cases in the "formal" trauma team system, suggesting that further research is warranted to investigate and attempt to replicate these findings in surgeon-led trauma systems.

In their study on the care of trauma patients that required hospital admission, Tuyp et al. have focused on the inpatient system of care and describe challenges and unique solutions that have evolved in British Columbia. ${ }^{3}$ Similar to Hayre et al., these authors highlight "outside the box" roles of emergency physicians with expanded inpatient responsibilities ranging from Level 1 referral hospitals to Level 3 centres. This study illustrates the value of diverse views, in addition to diverse skill sets, in the constant growth and quality improvement of regional trauma systems in Canada. Also, reflecting on the reality that local resources must be considered when developing trauma systems, they demonstrate the need to factor in sustainability when developing components of that system.

From *Trauma Nova Scotia, NS Department of Health and Wellness, Halifax, NS; †Department of Critical Care and $¥$ Department of Emergency Medicine, Dalhousie University, Halifax, NS; and the §University of Calgary Pediatric Surgery, Alberta Children's Hospital, Calgary, AB.

Correspondence to: Dr. Robert S. Green, Room 377, Bethune Building, 1276 South Park Street, Halifax, NS B3H 2Y9, Canada; Email: Robert. Green@nshealth.ca 
In the true Canadian spirit, health care in Canada strives for openness and collaboration as clinicians and other decision-makers realize that all have important roles in the development and evaluation of methods to benefit our trauma patient population. Indeed, previous studies have demonstrated that emergency physicians who perform the role of trauma team leader provide equivalent acute and resuscitative trauma care to their surgical colleagues. ${ }^{4}$ As highlighted in this issue of CFEM, both Hayre et al. ${ }^{1}$ and Tuyp et al. ${ }^{3}$ demonstrate how emergency physicians can and do play a central role in the care of trauma patients in Canada.

It should be viewed as a strength of Canadian trauma systems that emergency physicians can play equal leadership roles with their colleagues in all aspects of injury care, from prevention to resuscitation to quality assurance. "One size does not fit all." This inclusion of diverse strengths can only lead to truly functional models of care that "fit" local needs and resources, and that can achieve optimal outcomes for all Canadians.

Keywords: wounds, injuries, trauma systems

Competing interests: None declared.

\section{REFERENCES}

1. Hayre J, Rouse C, French J, et al. A traumatic tale of two cities: a comparison of outcomes for adults with major trauma who present to differing trauma centres in neighbouring Canadian provinces. CFEM 2018;20(2):191-9, doi: 10.1017/cem.2017.352.

2. Moore L, Evans D, Yanchar NL, et al. Canadian benchmarks for acute injury care. Can 7 Surg 2017;60(6):380-7.

3. Tuyp B, Hassani K, Constable L, Haegert J. Models of care for traumatically injured patients at trauma centres in British Columbia: variability and sustainability. C7EM 2018; 20(2):200-6, doi: 10.1017/cem.2017.50.

4. Ahmed JM, Tallon JM, Petrie DA. Trauma management outcomes associated with nonsurgeon versus surgeon trauma team leaders. Ann Emerg Med 2007;50(1):7-12. 